\title{
Alterations in the Occipital Cortex of Drug-Naïve Adults With Major Depressive Disorder: A Surface-Based Analysis of Surface Area and Cortical Thickness
}

\author{
Jee Soo Lee ${ }^{1}$, Wooyoung Kang ${ }^{2}$, Youbin Kang ${ }^{2}$, Aram Kim², \\ Kyu-Man Han ${ }^{1}$, Woo-Suk Tae ${ }^{3}$, and Byung-Joo Ham ${ }^{1 凶}$ \\ ${ }^{1}$ Department of Psychiatry, Korea University Anam Hospital, Korea University College of Medicine, Seoul, Republic of Korea \\ ${ }^{2}$ Department of Biomedical Sciences, Korea University College of Medicine, Seoul, Republic of Korea \\ ${ }^{3}$ Brain Convergence Research Center, Korea University Anam Hospital, Seoul, Republic of Korea
}

Objective Advances in surface-based morphometric methods have allowed researchers to separate cortical volume into cortical thickness (CTh) and surface area (SA). Although CTh alterations in major depressive disorder (MDD) have been observed in numerous studies, few studies have described significant SA alterations. Our study aimed to measure patients' SAs and to compare it with their CTh to examine whether SA exhibits alteration patterns that differ from those of CTh in drug-naïve patients with MDD.

Methods A total of 71 drug-naïve MDD patients and 111 healthy controls underwent structural magnetic resonance imaging, and SA and CTh were analyzed between the groups.

Results We found a smaller SA in the left superior occipital gyrus (L-SOG) in drug-naive patients with MDD. In the CTh analysis, the bilateral fusiform gyrus, left middle occipital gyrus, left temporal superior gyrus, and right posterior cingulate showed thinner cortices in patients with MDD, while the CTh of the bilateral SOG, right straight gyrus, right posterior cingulate, and left lingual gyrus were increased.

Conclusion Compared with the bilateral occipito-temporal changes in CTh, SA alterations in patients with MDD were confined to the L-SOG. These findings may improve our understanding of the neurobiological mechanisms of SA alteration in relation to MDD.

Psychiatry Investig 2021;18(1):1025-1033

Keywords Major depressive disorder; Surface area; Cortical thickness; Occipital cortex.

\section{INTRODUCTION}

Major depressive disorder (MDD) is one of the most disabling mental illnesses and a major public health concern. According to the recent global burden of disease study in 2017, the depressive disorder has prevailed as one of the leading causes of years lost to disability for the last three decades. ${ }^{1}$ However, the neurobiological mechanisms underlying MDD and its effects in the brain are still unclear.

Brain magnetic resonance imaging (MRI) has played a cru-

Received: March 16, 2021 Revised: June 27, 2021

Accepted: July 22, 2021

$\square$ Correspondence: Byung-Joo Ham, MD, PhD

Department of Psychiatry, Korea University Anam Hospital, Korea University College of Medicine, 73 Inchon-ro, Seongbuk-gu, Seoul 02841, Republic of Korea

Tel: +82-2-920-6843, Fax: +82-2-927-2836, E-mail: hambj@korea.ac.kr

(a) This is an Open Access article distributed under the terms of the Creative Commons Attribution Non-Commercial License (https://creativecommons.org/licenses/by$\mathrm{nc} / 4.0$ ) which permits unrestricted non-commercial use, distribution, and reproduction in any medium, provided the original work is properly cited. cial role in clarifying the structural abnormalities in MDD. ${ }^{2}$ Previous studies have traditionally focused on the cortical volume using voxel-based morphometry (VBM). However, VBM is limited in that it cannot distinguish between different cortical morphological properties. ${ }^{3}$ Advances in neuroimaging data processing have made it possible to divide the cortical volume into cortical thickness (CTh) and surface area (SA) using surface-based morphometry (SBM). These two morphometric parameters have been shown to be independent contributors to volume measurements and have distinct pathophysiological implications, with different genetic etiologies and developmental trajectories. ${ }^{4-7}$ Based on the hypothesis that neurons within the cerebral cortex are arrayed in columns perpendicular to the cortical surface, CTh represents the number of cells within columns, while SA reflects the number of cortical columns. ${ }^{8-11}$

The pattern of change in the CTh of patients with MDD has been reported in numerous studies, and recent meta-analyses provide strong evidence for the regional alteration of $\mathrm{CTh}$ in 
patients with MDD. ${ }^{12,13}$ Recent large meta- and mega-analyses of cortical abnormalities performed by the Enhancing Neuroimaging Genetics through Meta-Analysis (ENIGMA) neuroimaging consortium confirmed that patients with first-onset MDD exhibited significant cortical thinning in the fusiform gyrus, cingulate cortex, and insula, extending into the frontal cortex. ${ }^{14}$ On the other hand, SA in relation to MDD has not been studied sufficiently. Only a few studies with limited samples have reported significant changes in SA among drug-naive patients with MDD. The results of one previous research revealed that the SA of the left hippocampal gyrus increased in drugnaïve patients with MDD, based on the data of 46 patients, and another group of researchers found that the SA in subregions of the cingulate gyrus, parietal and frontal cortices could either be increased or decreased, based on the data of 16 patients. ${ }^{15,16}$ Although the ENIGMA consortium discovered that adolescent patients with recurrent MDD showed reduced SA in broad regions, they could not detect any significant differences in the SAs of adolescents with first-onset MDD and that of adults with first-onset and recurrent MDD. ${ }^{14}$

Despite the lack of research on the subject, SA is receiving more attention in relation to depression. During a recent analysis of the genetic correlation between cortical measurements and psychological traits, it was found that total SA, but not CTh, was negatively correlated with MDD and related psychological traits such as depressive symptoms, neuroticism, and insomnia. ${ }^{17}$ In another imaging-genetic study, researchers found that SA reduction in the occipito-temporal cluster was significantly associated with a high anxiety-depression score in twin samples. ${ }^{18}$ Such findings suggest that measuring SA is important when studying MDD. The pattern of SA alterations in patients with MDD needs to be studied further.

One strong point of our research is that we enrolled a relatively large number of drug-naïve adults with MDD from a single clinic to evaluate surface vulnerability in patients with MDD. Currently, this study on SA has a relatively large sample size ( $\mathrm{n}=$ 535) and follows from the ENIGMA meta-analysis research and analyzed the SA of drug-naïve patients with MDD. ${ }^{14}$ The exclusion of patients with MDD with a history of medication can reduce the potential influence of medication status and influence of chronic or recurrent episodes of MDD. ${ }^{19,20}$ We examined SA and CTh separately and analyzed their correlation with clinical variables. Our a priori hypothesis was that drug-naive patients with MDD would have alterations in their SAs that exhibit characteristic abnormalities that differ from those of CTh.

\section{METHODS}

\section{Participants}

The study included 71 patients with MDD and 111 healthy controls (HCs). Patients were recruited from the outpatient psychiatric clinic of Korea University Anam Hospital, located in Seoul, Republic of Korea, between February 2010 and December 2017. All MDD diagnoses were determined by board-certified psychiatrists (Ham BJ and Han KM) using the Structured Clinical Interview for DSM-IV Axis I Disorders. The patients included in the present study were adults aged 19-65 years. In addition, two psychiatrists assessed the duration of MDD by interviewing patients using the life-chart methodology. The exclusion criteria for this study were as follows; 1) primary or comorbid psychiatric diagnoses on Axis I or Axis II (based on DSM-IV-TR criteria) other than MDD; 2) MDD with psychotic features; 3 ) history of serious or unstable medical illness; 4) primary neurological illness; and 5) any contraindication for MRI, including pacemakers, metal implants, and claustrophobia. HC participants with no current or past history of psychiatric disorders were recruited using advertisements in the community. HCs were evaluated by two psychiatrists with the same set of exclusion criteria applied to the patient group. The age of healthy participants ranged from 19 to 65 years, and all participants were right-handed, as revealed by the Edinburgh Handedness Test. ${ }^{21}$ the severity of depressive symptoms for patients with MDD and HCs was measured using the 17-item Hamilton Depression Rating Scale (HDRS-17) on the day of MRI acquisition. ${ }^{22}$ The study protocol was approved by the Institutional Review Board of Korea University Anam Hospital (IRB No. 2009AN0105). All participants provided written informed consent to participate in the study in accordance with the Declaration of Helsinki (revised in 2008).

\section{Image acquisition}

T1-weighted images were acquired using a 3.0-Tesla Trio $^{\mathrm{TM}}$ whole-body imaging system (Siemens Healthcare GmbH, Erlangen, Germany). T1-weighted images were acquired parallel to the anterior-commissure-posterior-commissure line using the 3D T1-weighted magnetization-prepared rapid gradientecho sequence with the following parameters: repetition time, 1,900 ms; echo time, $2.6 \mathrm{~ms}$; the field of view, $220 \mathrm{~mm}$; matrix size, $256 \times 256$; slice thickness, $1 \mathrm{~mm}$; the number of coronal slices, 176 (without gap); voxel size, $0.86 \times 0.86 \times 1 \mathrm{~mm}^{3}$; flip angle, $16^{\circ}$ flip angle; and number of excitations, 1 .

\section{Image processing}

Analysis of CTh and SA was performed on the three-dimensional model of cortical surface reconstructions computed from T1 images using the FreeSurfer 6.0 version (Laboratory for Computational Neuroimaging, Athinoula A. Martinos Center for Biomedical Imaging, Charlestown, MA, USA; http:// surfer.nmr.mgh.harvard.edu). The details of the technical aspects of these procedures have been described in previous pub- 
lications. ${ }^{23-29}$ Briefly, the implanted processing stream involved motion correction of volumetric T1-weighted images, removal of non-brain tissue using a hybrid watershed/surface deformation procedure, automated Talairach transformation of each subject's native brain, segmentation of the gray matter-white matter volumetric structures, ${ }^{26}$ inflation of the cortical surface to an average spherical surface to locate both the pial surface and the gray matter-white matter boundary, intensity normalization, and automated topology correction. ${ }^{27}$ The transition of gray/white matter and the pial boundary was indicated by detecting the greatest shift in intensity through surface deformation.

We visually checked the cortical reconstruction of each subject for inaccuracies and manually corrected major topological inaccuracies with vertex edits or control points, and subsequently repeated the processing. The CTh was calculated as the shortest distance between the gray matter/white matter boundary and pial surface at each vertex across the cortical mantle, measured in millimeters ( $\mathrm{mm})$. Cortical SA was calculated as the sum of the area of the vertices falling within a given region on the white matter surface. Smoothing with a Gaussian kernel of $20 \mathrm{~mm}$ full width at half-maximum was performed on the cortical maps of each subject for the entire cortex analyses. ${ }^{28}$ In addition to vertex-based reconstruction, FreeSurfer automatically parcellated the cortex into 38 gyral-based regions-ofinterest per hemisphere, according to the Destrieux atlas. ${ }^{30}$ For each of the 76 cortical parcellations in the bilateral hemispheres, FreeSurfer calculates 1) the average CTh (in mm), 2) total cortical SA of the pial $\left(\mathrm{mm}^{2}\right)$.

\section{Statistical analysis}

Group differences in the demographic and clinical characteristics of the drug-naïve patients with MDD and HCs were analyzed using an independent $t$-test for continuous variables (age, TICV, illness duration, and HDRS scores) and chi-square tests for categorical variables (sex and education). All statistical analyses were performed using IBM SPSS Statistics for Windows (version 24.0; IBM Corp., Armonk, NY, USA).

As the main analysis, we compared CTh and SA between drug-naïve patients with MDD and HCs using a one-way analysis of covariance. The extracted values of CTh and SA in 76 cortical parcellations in the bilateral hemispheres were set as dependent variables and the groups (MDD vs. HC group) as independent variables. Age, sex, education level, and total intracranial volume (TICV), which was manually measured as previously described, were included as nuisance covariance in the analysis. ${ }^{31}$ For multiple comparisons, false discovery rate (FDR) correction, as described by Benjamini and Hochberg, ${ }^{32}$ was applied to each main analysis.

To determine the relationship between the mean values of $\mathrm{CTh}$ or SA and disease burden-related clinical characteristics (illness duration and severity of depression), a two-tailed Pearson's partial correlation analysis (adjusted for age, sex, education level, and TICV) was performed separately for both CTh and SA, which revealed significant differences between the $\mathrm{MDD}$ and $\mathrm{HC}$ groups in the main analysis.

\section{RESULTS}

\section{Demographic and genotypic characteristics}

We compared age, sex, education level, TICV, illness duration, and HDRS scores of drug-naïve patients with MDD and HCs. The results are presented in Table 1. The drug-naïve MDD and $\mathrm{HC}$ groups did not differ significantly in terms of age, sex, education level, and TICV. A significant difference was observed for the HDRS scores between diagnostic groups, with the mean (standard deviation) HDRS scores being 18.28 (4.99) and 1.53 (1.85) for patients and HCs, respectively ( $\mathrm{p}<0.001)$. The mean duration of illness in the patient group was $25.79 \pm 37.46$ months.

Table 1. Demographic and clinical characteristics of drug-naïve patients with major depressive disorder (MDD) and healthy controls (HCs)

\begin{tabular}{lccc}
\hline & Drug-naïve MDD patients (N=71) & HCs (N=111) & Significance (p-value) \\
\hline Age (years) & $40.97 \pm 12.43$ & $39.11 \pm 13.51$ & 0.350 \\
Sex (male/female) & $17 / 54$ & $38 / 73$ & 0.133 \\
Education level & & & 0.78 \\
$\quad$ Elementary and middle school & 15 & 16 & 83 \\
$\quad$ High school or college/university & 53 & 12 & 0.0219 \\
$\quad$ Above graduate school & 3 & $1452.26 \pm 167.03$ & N/A \\
TICV (cm ${ }^{3}$ ) & $1426.35 \pm 116.08$ & - & $<0.0001^{*}$ \\
Illness duration (months) & $25.79 \pm 37.46$ & $1.53 \pm 1.85$ & \\
HDRS score & $18.28 \pm 4.99$ & & \\
\hline
\end{tabular}

Significance was evaluated using a two-sample t-test. *denotes significance. TICV, total intracranial volume; HDRS, Hamilton Depression Rating Scale 


\section{Analysis of the differences in CTh between patients with MDD and HCs}

The mean CTh values of the cortical regions in the MDD and $\mathrm{HC}$ groups with their respective significant differences are shown in Table 2. Both significantly decreased and increased CTh values were observed in patients with MDD compared with those of $\mathrm{HCs}$ (at a statistical level of $\mathrm{p}<0.05$, FDR-corrected). The cortical regions with decreased thickness were the left and right fusiform gyri (left: $\mathrm{F}=8.621, \mathrm{p}_{\text {corr }}$ [FDR-corrected $\mathrm{p}$ value $=0.035$; right: $\mathrm{F}=8.456, \mathrm{p}_{\text {corr }}=0.035$ ), left middle occipital gyrus $\left(\mathrm{F}=8.847\right.$, $\left.\mathrm{p}_{\text {corr }}=0.035\right)$, right posterior-ventral part of cingulate gyrus $\left(\mathrm{F}=8.817, \mathrm{p}_{\text {corr }}=0.035\right)$, and left temporal plane of the superior temporal gyrus $\left(\mathrm{F}=9.087\right.$, $\left.\mathrm{p}_{\text {corr }}=0.035\right)$. Cortices with increased thickness in the patient group included the left and right superior occipital gyri (left: $F=22.311$, $\mathrm{p}_{\text {corr }}=0.00038$; right: $\mathrm{F}=12.132$, $\left.\mathrm{p}_{\text {corr }}=0.024\right)$, left lingual gyrus $\left(\mathrm{F}=10.175\right.$, $\mathrm{p}_{\text {corr }}=$ $0.035)$, right middle-posterior cingulate gyrus $\left(\mathrm{F}=8.124, \mathrm{p}_{\text {corr }}=\right.$ 0.037 ), and right straight gyrus ( $\left.\mathrm{F}=8.5, \mathrm{p}_{\text {corr }}=0.035\right)$. Correlation analysis performed for regions with a significant change in CTh did not reveal a significant correlation between changes in CTh and illness duration or HDRS scores (Table 3).

\section{Analysis of the difference in SA between patients with MDD and HCs}

As shown in Table 4, the mean SA of the left superior occipital gyrus (L-SOG) of drug-naïve patients with MDD was significantly smaller than that of HCs $\left(\mathrm{F}=14.87, \mathrm{p}_{\text {corr }}=0.012\right)$. Correlation analysis performed for the SA of the L-SOG did not

Table 2. Cortical regions with altered cortical thickness in drug-naïve patients with major depressive disorder (MDD), compared to healthy controls $(\mathrm{HC})$

\begin{tabular}{|c|c|c|c|c|}
\hline Cortical regions & MDD & $\mathrm{HC}$ & F-value & $\mathrm{p}_{\text {corr }}$ \\
\hline \multicolumn{5}{|l|}{$\mathrm{MDD}<\mathrm{HC}$} \\
\hline Lt. fusiform gyrus & $2.646 \pm 0.379$ & $2.795 \pm 0.283$ & 8.621 & $0.035^{*}$ \\
\hline Rt. fusiform gyrus & $2.663 \pm 0.331$ & $2.792 \pm 0.247$ & 8.456 & $0.035^{*}$ \\
\hline Lt. middle occipital gyrus & $2.499 \pm 0.22$ & $2.59 \pm 0.19$ & 8.847 & $0.035^{*}$ \\
\hline Rt. ventral part of the posterior cingulate gyrus & $2.456 \pm 0.374$ & $2.629 \pm 0.332$ & 8.817 & $0.035^{*}$ \\
\hline Lt. temporal plane of the superior temporal gyrus & $3.180 \pm 0.357$ & $3.335 \pm 0.322$ & 9.087 & $0.035^{*}$ \\
\hline \multicolumn{5}{|l|}{$\mathrm{MDD}>\mathrm{HC}$} \\
\hline Lt. superior occipital gyrus & $2.317 \pm 0.275$ & $2.144 \pm 0.202$ & 22.311 & $0.00038^{*}$ \\
\hline Rt. superior occipital gyrus & $2.297 \pm 0.276$ & $2.176 \pm 0.195$ & 12.132 & $0.024^{*}$ \\
\hline Lt. lingual gyrus & $2.085 \pm 0.423$ & $1.953 \pm 0.227$ & 10.175 & $0.035^{*}$ \\
\hline Rt. middle part of the posterior cingulate gyrus & $2.686 \pm 0.181$ & $2.612 \pm 0.162$ & 8.124 & $0.037^{*}$ \\
\hline Rt. straight gyrus & $2.743 \pm 0.348$ & $2.627 \pm 0.275$ & 8.5000 & $0.035^{*}$ \\
\hline
\end{tabular}

p $<0.05$ FDR-corrected. *denotes significance. Lt., left; Rt., right

Table 3. Correlation of the cortical thickness with the Hamilton Depression Rating Scale (HDRS) score or illness duration in patients with drug-naïve major depressive disorder (MDD)

\begin{tabular}{|c|c|c|c|c|}
\hline \multirow{2}{*}{ Cortical regions } & \multicolumn{2}{|c|}{ Illness duration } & \multicolumn{2}{|c|}{ HDRS } \\
\hline & $\mathrm{R}$ & $\mathrm{p}_{\text {corr }}$ & $\mathrm{R}$ & $\mathrm{p}_{\text {corr }}$ \\
\hline \multicolumn{5}{|l|}{$\mathrm{MDD}<\mathrm{HC}$} \\
\hline Lt. fusiform gyrus & -0.11422 & 0.82945 & -0.11657 & 0.54709 \\
\hline Rt. fusiform gyrus & -0.01064 & 0.93512 & -0.13365 & 0.54709 \\
\hline Lt. middle occipital gyrus & 0.06856 & 0.85657 & -0.05932 & 0.72194 \\
\hline Rt. posterior-ventral part of the cingulate gyrus & -0.01808 & 0.82945 & -0.09676 & 0.57276 \\
\hline Lt. temporal plane of the superior temporal gyrus & -0.15113 & 0.82945 & -0.12464 & 0.54709 \\
\hline \multicolumn{5}{|l|}{$\mathrm{MDD}>\mathrm{HC}$} \\
\hline Lt. superior occipital gyrus & 0.09289 & 0.82945 & -0.01547 & 0.90579 \\
\hline Rt. superior occipital gyrus & 0.08849 & 0.82945 & -0.13497 & 0.54709 \\
\hline Lt. lingual gyrus & 0.10703 & 0.82945 & -0.1137 & 0.54709 \\
\hline Rt. middle-posterior part of cingulate gyrus & 0.14985 & 0.93512 & -0.20272 & 0.54709 \\
\hline Rt. straight gyrus & -0.04283 & 0.92886 & -0.17116 & 0.54709 \\
\hline
\end{tabular}

p $<0.05$ FDR-corrected. HC, healthy controls; Lt., left; Rt., right 
Table 4. Cortical regions with altered surface area in drug-naïve patients with MDD, compared to HC

\begin{tabular}{lcccc}
\hline \multicolumn{1}{c}{ Cortical regions } & MDD & HC & F-value & p-value \\
\hline $\begin{array}{l}\text { MDD }<\text { HC } \\
\text { Lt. occipital superior gyrus }\end{array}$ & $921.89 \pm 123.09$ & $1028.78 \pm 205.97$ & 14.87 & $0.012^{*}$ \\
\hline
\end{tabular}

p $<0.05$ FDR-corrected. *denotes significance. MDD, major depressive disorder; HC, healthy controls; Lt., left

Table 5. Correlation of the cortical surface area with the Hamilton Depression Rating Scale (HDRS) score or illness duration in patients with drug-naïve major depressive disorder (MDD)

\begin{tabular}{lllll}
\hline \multirow{2}{*}{ Cortical regions } & \multicolumn{2}{c}{$\begin{array}{c}\text { Illness duration } \\
(\mathrm{mo})\end{array}$} & & \multicolumn{2}{l}{ HDRS } \\
\cline { 2 - 3 } & $\mathrm{R} \quad \mathrm{p}$-value & $\mathrm{R} \quad \mathrm{p}$-value \\
\hline
\end{tabular}

$\mathrm{MDD}<\mathrm{HC}$

$\begin{array}{lllll}\text { Lt. superior occipital } \quad 0.12887 & 0.29865 & 0.07791 & 0.53088\end{array}$

gyrus

$\mathrm{p}<0.05$. HC, healthy controls; mo, months; Lt., left

reveal a significant correlation between changes in SA and illness duration or HDRS scores (Table 5).

\section{Analysis of the correlation between CTh and SA in an altered region}

Since patients with MDD exhibited significant alterations in both the CTh and SA of their L-SOG, we conducted an additional two-tailed Pearson's partial correlation analysis to determine the relationship between CTh and SA in this region. We performed the correlation analysis separately for patients with MDD and HCs. Age, sex, education level, and TICV were controlled as covariates. As shown in Supplementary Table 1 (in the online-only Data Supplement), there was a significant positive correlation between the SA and CTh of L-SOG in the patient group $(\mathrm{R}=0.277, \mathrm{p}=0.023)$. In the $\mathrm{HC}$ group, $\mathrm{CTh}$ and $\mathrm{SA}$ of the L-SOG exhibited significant inverse correlations $(\mathrm{R}=$ $-0.271, \mathrm{p}=0.005$ ).

\section{DISCUSSION}

We examined cortical abnormalities in drug-naïve patients with MDD by simultaneously investigating CTh and SA. Our major finding was that in drug-naïe patients with MDD, CTh was altered to a broad extent, mostly affecting the bilateral occipitotemporal area, and SA was reduced in the L-SOG. To our knowledge, this is the first report of significant occipital SA alterations in drug-naïve adults with MDD.

\section{Cortical thickness}

The cortical regions with CTh alterations were similar to those observed in previous studies that analyzed CTh in drugnaïve patients with $\mathrm{MDD},{ }^{13,16,28,33,34}$ although we observed limited alteration of the occipitotemporal regions in our study. In contrast to previous studies, we could not replicate the commonly observed alterations in the prefrontal cortex. ${ }^{15,34-36}$

The results of our study showed that patients with MDD had a thinner cortex in the bilateral fusiform gyrus, left middle occipital gyrus (MOG), left superior temporal gyrus (STG), and right ventral posterior cingulate cortex (PCC). In contrast, cortical thickening was observed in the bilateral SOG, left lingual gyrus, right middle part of the PCC, and right straight gyrus in patients with MDD. Affected cortical regions are associated with visual perception and the default mode network. Several studies on MDD have observed cortical thinning in the fusiform gyrus, ${ }^{12,14,37}$ which plays a key role in distinguishing high-level visual information in face perception. ${ }^{38,39}$ Significant cortical alterations in the lingual gyrus, which is part of the visual association cortex, have also been observed in several studies on MDD. ${ }^{12,40,41}$ In the lateral occipital region, the medial, superior, and inferior occipital gyri are also mostly part of the visual association cortex and are observed to be vulnerable to depression. ${ }^{12,16,41-43}$ The STG is also associated with face processing and attention to emotion, and plays an important role in social and emotional processing. ${ }^{44-46}$ Such findings suggest that changes in the occipitotemporal region may manifest as clinical symptoms of MDD, such as an impairment in social perception. The PCC is a core node in the default mode network, which is associated with cognitive control and self-referential thinking ${ }^{47}$ and is frequently disrupted in patients with MDD. ${ }^{12,13,48,49}$ Additionally, the PCC is a region that is functionally connected to the occipital region and is de-activated when the lateral occipital cortex is activated. ${ }^{50}$

The reason for the complex pattern of regional hypertrophic and atrophic cortical changes in our study is unclear. However, the results of previous studies on patients with untreated MDD have often indicated both regional increases and decreases in CTh. ${ }^{15,48}$ A possible explanation for the pathophysiology of cortical thickening is that it is caused by cellular changes resulting from reactive gliosis or neurogenesis in the early course of MDD to recover from insult. ${ }^{51,52}$ In contrast, cortical thinning may occur due to neurotoxic and/or gliotoxic processes. ${ }^{53,54}$ The heterogeneous pattern of functional dysconnectivity and differing symptom combinations within the MDD group may have contributed to this divergent pattern. Factors that determine the type of change in the CTh need to be examined in future research. 


\section{Surface area}

Although both CTh and SA indicated cortical changes in the occipital lobe, SA showed a finer result, restricted to the LSOG. This is one of the few studies to report a significant reduction of SA in drug-naïve patients with MDD to the best of our knowledge. ${ }^{15,16}$ This is the first report of SA reduction in the SOG in MDD. Nevertheless, SA reduction in the occipital gyrus has been reported in several subgroups of MDD. A recent SBM study revealed that patients with current episodes of MDD showed increased SA in the left precuneus and right pericalcarine gyrus compared to remitted MDD ${ }^{55}$ Moreover, one twin study demonstrated widespread SA reduction in an occipitotemporal cluster in subjects with high anxiety-depression score. ${ }^{18}$ Our finding is partly supported by previous research that showed larger SA in the left lateral occipital cortex and postcentral area in MDD patients with a history of suicide attempt(s) compared to non-attempters. ${ }^{56}$ However, our sample of drug-naïve patients with MDD showed a reduction, not an enlargement of the SA.

In our study, the average L-SOG SA decreased while the average CTh in the same region increased. This inverse change has been observed in both HC and patient groups in several studies. ${ }^{5,57-60}$ According to the "balloon model" hypothesis, the cortex is stretched out tangentially to the pial surface as a result of white matter myelin growth like a balloon, resulting in a larger SA and lower $\mathrm{CTh}^{61-63}$ The results of the correlation analysis between the patient and $\mathrm{HC}$ groups revealed that $\mathrm{HCs}$ exhibited a significant inverse correlation between CTh and SA, supporting the balloon model hypothesis $(\mathrm{R}=-0.271, \mathrm{p}=0.005)$. However, a positive correlation was found within the patient group $(\mathrm{R}=0.277, \mathrm{p}=0.023)$. We speculate that the reduction of SA caused by a disease entity cannot be solely caused by the concomitant process of cortical thickening. Different patterns of correlation between the two parameters in healthy subjects and the patient group need to be analyzed in future studies.

There are plausible explanations for why the L-SOG is specifically vulnerable to $S A$ reduction. First, this could be due to low gamma-aminobutyric acid (GABA) concentration in the occipital cortex, which is commonly observed in MDD. ${ }^{64-68}$ The occipital lobe exhibits a high expression of $\mathrm{GABA},{ }^{69,70}$ and genes that modulate GABAergic transmission such as $\mathrm{BDNF}^{71,72}$ and GAD67 $7^{73,74}$ are subject to epigenetic changes in MDD. A positive correlation between the GABA concentration and SA size of the primary visual cortex was observed ${ }^{75}$ supporting our hypothesis that alteration of GABAergic neurotransmission in MDD might lead to SA reduction. Second, the L-SOG has the potential to be an important node in the visual network. Biophysical limitations during SA alteration can cause network disruption. ${ }^{55,76}$ The SOG is functionally connected to other regions of the occipital lobe and PCC, comprising the lateral vi- sual cortical network. ${ }^{50,77,78}$ The adjacent L-MOG has shown decreased activity in several MDD studies, ${ }^{79-81}$ and left inferior longitudinal fasciculus, a white matter tract that connects the SOG to the limbic system, ${ }^{50,82}$ showed disrupted integrity in drug-naïve MDD. ${ }^{83}$ Third, the SA of the L-SOG could be more susceptible to genetic factors related to MDD. SA has higher heritability than $C \mathrm{Ch}^{17,84,85}$ and the occipital cortex has been reported to be the region with the highest heritability among the cerebral cortex, especially in relation to MDD. ${ }^{18,86}$ This implies that SA alteration in the L-SOG could be a genetically determined feature that contributes to the etiology of MDD.

\section{Correlations with illness duration and symptom severity}

We did not detect a significant correlation between cortical alteration and illness duration or the HDRS score. This is consistent with the findings of the ENIGMA consortium, implying that cortical measurements do not directly represent the clinical state. ${ }^{14}$ However, a few studies have reported SA differences according to remission state of MDD or in relation to the anxiety-depression score..$^{18,55}$ Therefore, further studies are needed to clarify this issue.

\section{Limitation}

Our study had some limitations. First, the cross-sectional design of this study meant that we could not determine whether cortical alteration is a causative or secondary phenomenon. Longitudinal data are required to understand causal relationships. Second, our analysis was restricted to cortical regions, and measurements of subcortical regions such as the thalamus, hippocampus, amygdala, and striatum were not included. As both cortical and subcortical regions are reportedly involved in MDD, analyzing both the cortical and subcortical areas separately may lead to more precise results. Third, we could not replicate the previously reported alterations in the prefrontal cortex of patients with MDD. This may be due to the heterogeneity of the sample or limitations of the atlas-based method. Considering that most of the studies that reported significant changes in CTh in prefrontal regions used vertex-based analyses and compared CTh values at each vertex, ${ }^{28,42,87}$ our method of comparing the mean values of the functionally predefined regions may have averaged out the complex pattern of deformity within the region. Furthermore, relatively small sample size may contribute to null finding regarding the prefrontal cortex. In future studies, combining the use of vertex-based comparison and atlas-based comparison would strengthen the evidence gathered. Integration of anatomical data with functional imaging data or genetic studies in the future may shed light on the neural mechanisms underlying SA alterations in the occipital cortex. 
In conclusion, our results indicated that drug-naïve patients with MDD had a smaller L-SOG SA. Along with the results of the CTh analysis, we found that patients with MDD are predisposed to alterations in the occipital area. Furthermore, the physiological, functional, and genetic traits of the occipital cortex may explain why SA alterations are more likely to occur in the L-SOG. Further studies are required to determine the role of SA in MDD.

\section{Supplementary Materials}

The online-only Data Supplement is available with this article at https://doi.org/10.30773/pi.2021.0099.

\section{Availability of Data and Material}

All data generated or analyzed during the study are included in this published article (and its supplementary information files).

\section{Conflicts of Interest}

Kyu-Man Han, a contributing editor of the Psychiatry Investigation, was not involved in the editorial evaluation or decision to publish this article. All remaining authors have declared no conflicts of interest.

\section{Author Contributions}

Conceptualization: Byung-Joo Ham. Data curation: Wooyoung Kang, Youbin Kang, Aram Kim. Formal analysis: Kyu-Man Han, Woo-Suk Tae. Funding acquisition: Byung-Joo Ham. Investigation: Byung-Joo Ham. Methodology: Kyu-Man Han, Woo-Suk Tae. Project administration: Byung-Joo Ham. Resources: Byung-Joo Ham. Software: Woo-Suk Tae. Supervision: Byung-Joo Ham. Validation: Kyu-Man Han. Visualization: KyuMan Han. Writing_original draft: Jee Soo Lee. Writing-review \& editing: Byung-Joo Ham, Kyu-Man Han.

\section{ORCID iDs}

Jee Soo Lee

Wooyoung Kang

Youbin Kang

Aram Kim

Kyu-Man Han

Woo-Suk Tae

Byung-Joo Ham

https://orcid.org/0000-0003-2558-8069

https://orcid.org/0000-0003-4733-027X

https://orcid.org/0000-0002-6173-9920

https://orcid.org/0000-0002-3941-218X

https://orcid.org/0000-0002-1982-4216

https://orcid.org/0000-0003-0451-0713

https://orcid.org/0000-0002-0108-2058

\section{Funding Statement}

This work was supported by the National Research Foundation of Korea (NRF) funded by the Ministry of Education, Science and Technology (NRF-2020M3E5D9080792).

\section{REFERENCES}

1. GBD 2017 Disease and Injury Incidence and Prevalence Collaborators. Global, regional, and national incidence, prevalence, and years lived with disability for 354 diseases and injuries for 195 countries and territories, 1990-2017: a systematic analysis for the Global Burden of Disease Study 2017. Lancet Lond Engl 2018 10;392:1789-1858.

2. Qiu H, Li J. Major depressive disorder and magnetic resonance imaging: a mini-review of recent progress. Curr Pharm Des 2018;24:25242529 .

3. Ashburner J, Friston KJ. Why voxel-based morphometry should be used. Neuroimage 2001;14:1238-1243.

4. Panizzon MS, Fennema-Notestine C, Eyler LT, Jernigan TL, PromWormley E, Neale M, et al. Distinct genetic influences on cortical surface area and cortical thickness. Cereb Cortex 2009;19:2728-2735.
5. Storsve AB, Fjell AM, Tamnes CK, Westlye LT, Overbye K, Aasland HW, et al. Differential longitudinal changes in cortical thickness, surface area and volume across the adult life span: regions of accelerating and decelerating change. J Neurosci Off J Soc Neurosci 2014;34:84888498 .

6. Wierenga LM, Langen M, Oranje B, Durston S. Unique developmental trajectories of cortical thickness and surface area. Neuroimage 2014; 87:120-126.

7. Tamnes CK, Herting MM, Goddings AL, Meuwese R, Blakemore SJ, Dahl RE, et al. Development of the cerebral cortex across adolescence: a multisample study of inter-related longitudinal changes in cortical volume, surface area, and thickness. J Neurosci Off J Soc Neurosci 2017; 37:3402-3412.

8. Mountcastle VB. The columnar organization of the neocortex. Brain J Neurol 1997;120:701-722.

9. Rakic P. Defects of neuronal migration and the pathogenesis of cortical malformations. Prog Brain Res 1988;73:15-37.

10. Rakic P. A small step for the cell, a giant leap for mankind: a hypothesis of neocortical expansion during evolution. Trends Neurosci 1995; 18:383-388.

11. Rakic P. The radial edifice of cortical architecture: from neuronal silhouettes to genetic engineering. Brain Res Rev 2007;55:204-219.

12. Suh JS, Schneider MA, Minuzzi L, MacQueen GM, Strother SC, Kennedy SH, et al. Cortical thickness in major depressive disorder: a systematic review and meta-analysis. Prog Neuropsychopharmacol Biol Psychiatry 2019 10;88:287-302.

13. Li Q, Zhao Y, Chen Z, Long J, Dai J, Huang X, et al. Meta-analysis of cortical thickness abnormalities in medication-free patients with major depressive disorder. Neuropsychopharmacology 2020;45:703-712.

14. Schmaal L, Hibar DP, Sämann PG, Hall GB, Baune BT, Jahanshad N, et al. Cortical abnormalities in adults and adolescents with major depression based on brain scans from 20 cohorts worldwide in the ENIGMA Major Depressive Disorder Working Group. Mol Psychiatry 2017;22:900-909.

15. Qiu L, Lui S, Kuang W, Huang X, Li J, Li J, et al. Regional increases of cortical thickness in untreated, first-episode major depressive disorder. Transl Psychiatry 2014;4:e378.

16. Peng D, Shi F, Li G, Fralick D, Shen T, Qiu M, et al. Surface vulnerability of cerebral cortex to major depressive disorder. PLos One 2015;10: e0120704.

17. Grasby KL, Jahanshad N, Painter JN, Colodro-Conde L, Bralten J, Hibar DP, et al. The genetic architecture of the human cerebral cortex. Science 2020;367:eaay6690.

18. Couvy-Duchesne B, Strike LT, Zubicaray GI de, McMahon KL, Thompson PM, Hickie IB, et al. Lingual Gyrus Surface Area Is Associated with Anxiety-Depression Severity in Young Adults: A Genetic Clustering Approach. eNeuro [Internet]. Available at: https://www.eneuro.org/ content/5/1/ENEURO.0153-17.2017. Accessed Jan 13, 2021.

19. Koolschijn PC, van Haren NE, Lensvelt-Mulders GJ, Hulshoff Pol HE, Kahn RS. Brain volume abnormalities in major depressive disorder: a meta-analysis of magnetic resonance imaging studies. Hum Brain Mapp 2009;30:3719-3735.

20. Du MY, Wu QZ, Yue Q, Li J, Liao Y, Kuang WH, et al. Voxelwise metaanalysis of gray matter reduction in major depressive disorder. Prog Neuropsychopharmacol Biol Psychiatry 2012;36:11-16.

21. Oldfield RC. The assessment and analysis of handedness: the Edinburgh inventory. Neuropsychologia 1971;9:97-113.

22. Hamilton M. A rating scale for depression. J Neurol Neurosurg Psychiatry 1960;23:56-62.

23. Dale AM, Fischl B, Sereno MI. Cortical surface-based analysis. I. Segmentation and surface reconstruction. Neuroimage 1999;9:179-194.

24. Fischl B, Liu A, Dale AM. Automated manifold surgery: constructing geometrically accurate and topologically correct models of the human cerebral cortex. IEEE Trans Med Imaging 2001;20:70-80.

25. Fischl B, Salat DH, Busa E, Albert M, Dieterich M, Haselgrove C, et al. 
Whole brain segmentation: automated labeling of neuroanatomical structures in the human brain. Neuron 2002;33:341-355.

26. Fischl B, van der Kouwe A, Destrieux C, Halgren E, Ségonne F, Salat $\mathrm{DH}$, et al. Automatically parcellating the human cerebral cortex. Cereb Cortex 2004;14:11-22.

27. Ségonne F, Pacheco J, Fischl B. Geometrically accurate topology-correction of cortical surfaces using nonseparating loops. IEEE Trans Med Imaging 2007;26:518-529.

28. Han KM, Choi S, Jung J, Na KS, Yoon HK, Lee MS, et al. Cortical thickness, cortical and subcortical volume, and white matter integrity in patients with their first episode of major depression. J Affect Disord 2014; 155:42-48.

29. Han KM, Won E, Sim Y, Kang J, Han C, Kim YK, et al. Influence of FKBP5 polymorphism and DNA methylation on structural changes of the brain in major depressive disorder. Sci Rep 2017;7:42621.

30. Destrieux C, Fischl B, Dale A, Halgren E. Automatic parcellation of human cortical gyri and sulci using standard anatomical nomenclature. Neuroimage 2010;53:1-15.

31. Tae WS, Kim SS, Lee KU, Nam EC, Kim KW. Validation of hippocampal volumes measured using a manual method and two automated methods (FreeSurfer and IBASPM) in chronic major depressive disorder. Neuroradiology 2008;50:569-581.

32. Benjamini $Y$, Hochberg Y. Controlling the false discovery rate: a practical and powerful approach to multiple testing. J R Stat Soc Ser B Methodol 1995;57:289-300.

33. Liu X, Kakeda S, Watanabe K, Yoshimura R, Abe O, Ide S, et al. Relationship between the cortical thickness and serum cortisol levels in drug-naïve, first-episode patients with major depressive disorder: a surface-based morphometric study. Depress Anxiety 2015;32:702-708.

34. Kakeda S, Watanabe K, Katsuki A, Sugimoto K, Igata N, Ueda I, et al. Relationship between interleukin (IL)- 6 and brain morphology in drugnaïve, first-episode major depressive disorder using surface-based morphometry. Sci Rep 2018;8:10054.

35. Grieve SM, Korgaonkar MS, Koslow SH, Gordon E, Williams LM. Widespread reductions in gray matter volume in depression. Neuroimage Clin 2013;3:332-339.

36. Späti J, Hänggi J, Doerig N, Ernst J, Sambataro F, Brakowski J, et al. Prefrontal thinning affects functional connectivity and regional homogeneity of the anterior cingulate cortex in depression. Neuropsychopharmacology 2015;40:1640-1648.

37. Papmeyer M, Giles S, Sussmann JE, Kielty S, Stewart T, Lawrie SM, et al. Cortical thickness in individuals at high familial risk of mood disorders as they develop major depressive disorder. Biol Psychiatry 2015; 78:58-66.

38. Kanwisher N, Yovel G. The fusiform face area: a cortical region specialized for the perception of faces. Philos Trans R Soc Lond B Biol Sci 2006;361:2109-2128.

39. Tsantani M, Kriegeskorte N, Storrs K, Williams AL, McGettigan C, Garrido L. FFA and OFA encode distinct types of face identity information. J Neurosci Off J Soc Neurosci 2021;41:1952-1969.

40. Spang K, Grimsen C, Prass M, Brunner F, Köhnlein M, Kehrer S, et al. Midlevel visual deficits after strokes involving area human V4. Cortex J Devoted Study Nerv Syst Behav 2020;134:207-222.

41. Na KS, Won E, Kang J, Chang HS, Yoon HK, Tae WS, et al. Brain-derived neurotrophic factor promoter methylation and cortical thickness in recurrent major depressive disorder. Sci Rep 2016;6:21089.

42. Tu PC, Chen LF, Hsieh JC, Bai YM, Li CT, Su TP. Regional cortical thinning in patients with major depressive disorder: a surface-based morphometry study. Psychiatry Res 2012;202:206-213.

43. Kim JH, Suh S, Lee HJ, Lee J, Lee MS. Cortical and subcortical gray matter alterations in first-episode drug-naïve adolescents with major depressive disorder: Neuroreport 2019;30:1172-1178.

44. Morris JS, Friston KJ, Büchel C, Frith CD, Young AW, Calder AJ, et al. A neuromodulatory role for the human amygdala in processing emotional facial expressions. Brain J Neurol 1998;121:47-57.
45. Ellison A, Schindler I, Pattison LL, Milner AD. An exploration of the role of the superior temporal gyrus in visual search and spatial perception using TMS. Brain J Neurol 2004;127:2307-2315.

46. Narumoto J, Okada T, Sadato N, Fukui K, Yonekura Y. Attention to emotion modulates fMRI activity in human right superior temporal sulcus. Brain Res Cogn Brain Res 2001;12:225-231.

47. Fransson P, Marrelec G. The precuneus/posterior cingulate cortex plays a pivotal role in the default mode network: evidence from a partial correlation network analysis. Neuroimage 2008;42:1178-1184.

48. van Eijndhoven P, van Wingen G, Katzenbauer M, Groen W, Tepest R, Fernández G, et al. Paralimbic cortical thickness in first-episode depression: evidence for trait-related differences in mood regulation. Am J Psychiatry 2013;170:1477-1486.

49. van Tol MJ, Li M, Metzger CD, Hailla N, Horn DI, Li W, et al. Local cortical thinning links to resting-state disconnectivity in major depressive disorder. Psychol Med 2014;44:2053-2065.

50. Thiebaut de Schotten M, Urbanski M, Valabregue R, Bayle DJ, Volle E. Subdivision of the occipital lobes: an anatomical and functional MRI connectivity study. Cortex 2014;56:121-137.

51. Gould E, Reeves AJ, Graziano MSA, Gross CG. Neurogenesis in the neocortex of adult primates. Science 1999;286:548-552.

52. Ohira K. Injury-induced neurogenesis in the mammalian forebrain. Cell Mol Life Sci 2011;68:1645-1656.

53. Rajkowska G. Postmortem studies in mood disorders indicate altered numbers of neurons and glial cells. Biol Psychiatry 2000;48:766-777.

54. Sorrells SF, Munhoz CD, Manley NC, Yen S, Sapolsky RM. Glucocorticoids increase excitotoxic injury and inflammation in the hippocampus of adult male rats. Neuroendocrinology 2014;100:129-140.

55. Xiong G, Dong D, Cheng C, Jiang Y, Sun X, He J, et al. State-independent and -dependent structural alterations in limbic-cortical regions in patients with current and remitted depression. J Affect Disord 2019; 258:1-10.

56. Kang SG, Cho SE, Na KS, Lee JS, Joo SW, Cho SJ, et al. Differences in brain surface area and cortical volume between suicide attempters and non-attempters with major depressive disorder. Psychiatry Res Neuroimaging 2020;297:111032. [Online ahead of print]

57. Hogstrom LJ, Westlye LT, Walhovd KB, Fjell AM. The structure of the cerebral cortex across adult life: age-related patterns of surface area, thickness, and gyrification. Cereb Cortex 2013;23:2521-2530.

58. Bois C, Ronan L, Levita L, Whalley HC, Giles S, McIntosh AM, et al. Cortical surface area differentiates familial high risk individuals who go on to develop schizophrenia. Biol Psychiatry 2015;78:413-420.

59. Lee NR, Adeyemi EI, Lin A, Clasen LS, Lalonde FM, Condon E, et al. Dissociations in cortical morphometry in youth with down syndrome: evidence for reduced surface area but increased thickness. Cereb Cortex 2016;26:2982-2990.

60. Zhang X, Luo Q, Wang S, Qiu L, Pan N, Kuang W, et al. Dissociations in cortical thickness and surface area in non-comorbid never-treated patients with social anxiety disorder. EBioMedicine [Internet]. Available at: https://www.thelancet.com/journals/ebiom/article/PIIS23523964(20)30285-1/abstract. Accessed Jan 14, 2021.

61. Harasty J, Seldon HL, Chan P, Halliday G, Harding A. The left human speech-processing cortex is thinner but longer than the right. Laterality $2003 ; 8: 247-260$.

62. Seldon HL. Cortical laminar thickness and column spacing in human temporal and inferior parietal lobes: intra-individual anatomical relations. Laterality 2006;11:226-250.

63. Seldon HL. Does brain white matter growth expand the cortex like a balloon? Hypothesis and consequences. Laterality 2005;10:81-95.

64. Hasler G, van der Veen JW, Tumonis T, Meyers N, Shen J, Drevets WC. Reduced prefrontal glutamate/glutamine and gamma-aminobutyric acid levels in major depression determined using proton magnetic resonance spectroscopy. Arch Gen Psychiatry 2007;64:193-200.

65. Luscher B, Fuchs T. GABAergic control of depression-related brain states. Adv Pharmacol 2015;73:97-144. 
66. Maciag D, Hughes J, O’Dwyer G, Pride Y, Stockmeier CA, Sanacora G, et al. Reduced density of calbindin immunoreactive GABAergic neurons in the occipital cortex in major depression: relevance to neuroimaging studies. Biol Psychiatry 2010;67:465-470.

67. Sanacora G, Gueorguieva R, Epperson CN, Wu YT, Appel M, Rothman DL, et al. Subtype-specific alterations of gamma-aminobutyric acid and glutamate in patients with major depression. Arch Gen Psychiatry 2004;61:705-713.

68. Sanacora G, Mason GF, Rothman DL, Krystal JH. Increased occipital cortex GABA concentrations in depressed patients after therapy with selective serotonin reuptake inhibitors. Am J Psychiatry 2002;159:663665.

69. Meinecke DL, Rakic P. Expression of GABA and GABAA receptors by neurons of the subplate zone in developing primate occipital cortex: evidence for transient local circuits. J Comp Neurol 1992;317:91-101.

70. Wen L, Liao C, Chen M, Zhu D, Yan X, Zhong J, et al. Detection and Evaluation of GABA Concentration in Anterior Cingulate Cortex and Occipital Cortex by MEGA-PRESS. In: 2015 7th International Conference on Information Technology in Medicine and Education (ITME) [Internet]. Huangshan, China: IEEE; 2015, p. 80-84. Available at: http:// ieeexplore.ieee.org/document/7429102/. Accessed Jan 29, 2021.

71. D’Addario C, Dell'Osso B, Galimberti D, Palazzo MC, Benatti B, Di Francesco A, et al. Epigenetic modulation of BDNF gene in patients with major depressive disorder. Biol Psychiatry 2013;73:e6-e7.

72. Karpova NN. Role of BDNF epigenetics in activity-dependent neuronal plasticity. Neuropharmacology 2014;76:709-718.

73. Dong E, Agis-Balboa RC, Simonini MV, Grayson DR, Costa E, Guidotti A. Reelin and glutamic acid decarboxylase67 promoter remodeling in an epigenetic methionine-induced mouse model of schizophrenia. Proc Natl Acad Sci U S A 2005;102:12578-12583.

74. Zhang TY, Hellstrom IC, Bagot RC, Wen X, Diorio J, Meaney MJ. Maternal care and DNA methylation of a glutamic acid decarboxylase 1 promoter in rat hippocampus. J Neurosci Off J Soc Neurosci 2010;30: 13130-13137.

75. Bergmann J, Pilatus U, Genç E, Kohler A, Singer W, Pearson J. V1 surface size predicts GABA concentration in medial occipital cortex. NeuroImage 2016;124:654-662.

76. Kanai R, Rees G. The structural basis of inter-individual differences in human behaviour and cognition. Nat Rev Neurosci 2011;12:231-242.

77. Beckmann CF, DeLuca M, Devlin JT, Smith SM. Investigations into resting-state connectivity using independent component analysis. Philos Trans R Soc Lond B Biol Sci 2005;360:1001-1013.

78. Smith SM, Fox PT, Miller KL, Glahn DC, Fox PM, Mackay CE, et al. Correspondence of the brain's functional architecture during activation and rest. Proc Natl Acad Sci U S A 2009;106:13040-13045.

79. Fan Q, Palaniyappan L, Tan L, Wang J, Wang X, Li C, et al. Surface anatomical profile of the cerebral cortex in obsessive-compulsive disorder: a study of cortical thickness, folding and surface area. Psychol Med 2013;43:1081-1091.

80. Teng C, Zhou J, Ma H, Tan Y, Wu X, Guan C, et al. Abnormal resting state activity of left middle occipital gyrus and its functional connectivity in female patients with major depressive disorder. BMC Psychiatry 2018;18:370.

81. Chen Z, Peng W, Sun H, Kuang W, Li W, Jia Z, et al. High-field magnetic resonance imaging of structural alterations in first-episode, drugnaive patients with major depressive disorder. Transl Psychiatry 2016; 6:e942.

82. Liao Y, Huang X, Wu Q, Yang C, Kuang W, Du M, et al. Is depression a disconnection syndrome? Meta-analysis of diffusion tensor imaging studies in patients with MDD. J Psychiatry Neurosci JPN 2013;38:4956.

83. Won E, Kang J, Choi S, Kim A, Han KM, Yoon HK, et al. The association between substance $\mathrm{P}$ and white matter integrity in medicationnaive patients with major depressive disorder. Sci Rep 2017;7:9707.

84. Winkler AM, Kochunov P, Blangero J, Almasy L, Zilles K, Fox PT, et al. Cortical thickness or grey matter volume? The importance of selecting the phenotype for imaging genetics studies. Neuroimage 2010;53:11351146 .

85. Fjell AM, Chen CH, Sederevicius D, Sneve MH, Grydeland H, Krogsrud SK, et al. Continuity and discontinuity in human cortical development and change from embryonic stages to old age. Cereb Cortex 2019; 29:3879-3890.

86. Patel S, Patel R, Park MTM, Masellis M, Knight J, Chakravarty MM. Heritability estimates of cortical anatomy: the influence and reliability of different estimation strategies. Neuroimage 2018;178:78-91.

87. Järnum H, Eskildsen SF, Steffensen EG, Lundbye-Christensen S, Simonsen CW, Thomsen IS, et al. Longitudinal MRI study of cortical thickness, perfusion, and metabolite levels in major depressive disorder. Acta Psychiatr Scand 2011;124:435-446. 
Supplementary Table 1. Correlation between the cortical surface area and the cortical thickness among patients with drug-naïve major depressive disorder (MDD) and healthy controls (HC)

\begin{tabular}{lll}
\hline \multicolumn{1}{c}{ Cortical region } & $\mathrm{R}$ & $\mathrm{p}$-value \\
\hline MDD & & \\
$\quad$ Left superior occipital gyrus & 0.277 & $0.023^{*}$ \\
$\mathrm{HC}$ & & \\
$\quad$ Left superior occipital gyrus & -0.271 & $0.005^{*}$ \\
\hline $\mathrm{p}<0.05 . *$ denotes significance & &
\end{tabular}

